04,06

\title{
Потенциальные изображения сегнетоэлектрических доменных структур в кристаллах ниобата лития после формирования электронным лучом
}

\author{
(C) Л.С. Коханчик \\ Институт проблем технологии микроэлектроники и особочистых материалов РАН, \\ Черноголовка, Московская обл., Россия \\ E-mail: mlk@iptm.ru
}

(Поступила в Редакцию 21 фревраля 2018 г.)

\begin{abstract}
В кристаллах ниобата лития с помощью низковольтной SEM-микроскопии исследовались сегнетоэлектрические доменные структуры, созданные электронным лучом. Структуры были сформированы в кристаллах с различной проводимостью - в высокоомных образцах конгруэнтного состава (CLN) и в образцах с проводимостью, улучшенной благодаря восстановительному отжигу (RLN). Проанализирована потенциальная природа контраста доменных структур, наблюдаемых в режиме вторичных электронов в зависимости от проводимости образцов и направления спонтанной поляризации доменов. Предполагается, что контраст доменов в кристаллах CLN связан с длительно сохраняющимися зарядами, локализованными у доменных стенок и в зонах облучения. Записанные доменные структуры в кристаллах CLN визуализировались на полярных и неполярных срезах. В кристаллах RLN с улучшенной, по сравнению с CLN, проводимостью потенциальный контраст периодических доменных структур был обнаружен только на полярных срезах, где вектор $P_{s}$ доменов перпендикулярен облучаемой поверхности. Этот контраст, по-видимому, связан с влиянием поля зарядов спонтанной электрической поляризации на вторичные электроны.
\end{abstract}

Работа выполнена в рамках государственного задания № 007-00220-18-00.

DOI: 10.21883/FTT.2018.09.46391.046

\section{1. Введение}

Метаниобат лития $\mathrm{LiNbO}_{3}$ (LN) благодаря уникальному набору важных свойств относится к числу материалов, перспективных для разнообразных практических применений, в том числе для нелинейной и интегральной оптики [1]. В этой связи весьма актуальной является проблема преобразования частоты оптического излучения, которая может быть решена, в частности с использованием сегнетоэлектрической доменной структуры в качестве нелинейной среды. Развитие методов „доменной инженерии“ для LN, поиск путей создания периодических структур разного типа с субмикронными и микронными периодами расширяет возможности использования этих кристаллов. Создание доменов электронным лучом (DEBW-domain electron beam writing) является одним из методов доменной инженерии, позволяющим бесконтактное формирование довольно крупных участков периодически поляризованного ниобата лития (PPLN) в массивных и в пленочных образцах, а также в волноводных структурах на LN [2-10].

Развитие доменной инженерии требует использования разнообразных методов исследования PPLN. Некоторые методики диагностики доменов описаны, например, в обзорах [11,12]. Данная работа посвящена рассмотрению возможностей низковольтной растровой электронной микроскопии (SEM) для характеризации доменов, созданных методом DEBW в кристаллах LN.

Известно, что при низких ускоряющих напряжениях в режиме вторичных электронов (SE) имеется воз- можность наблюдать и изучать сегнетоэлектрические домены без их химического травления или декорирования поверхности [13-22]. В обзоре [13] представлены результаты наиболее ранних исследований равновесных доменных структур с использованием низковольтной SEM-микроскопии. Однако природа возникающего изображения доменов при сканировании электронным лучом по поверхности многих сегнетоэлектрических кристаллов до сих пор является предметом дискуссий. Авторы $[14,15]$ связывают SE-контраст $180^{\circ}$-х доменов с влиянием спонтанной электрической поляризации $\left(P_{s}\right)$ на количество детектируемых SE с поверхности доменов разного знака. В $[16,17]$ похожий контраст $180^{\circ}$-х доменов объяснялся небольшим нагревом поверхности из-за облучения электронами и возникновением на их поверхности пироэлектрических зарядов, также приводящих к контрасту SE. В работах [18-21] отмечено, что контраст доменных границ и контраст доменов часто возникает при небольшой зарядке плохо проводящих сегнетоэлектрических образцов. Противоречивость в обсуждениях природы SE-изображений доменов подчеркнута в [22,23], где предполагается одновременное влияние нескольких факторов на получаемые в SEM изображения доменов.

B настоящей работе обсуждаются SE-изображения доменных структур, созданных электронным лучом в кристаллах LN. Анализируются причины возникновения контраста доменов после их записи на полярных (001) и неполярных (010) поверхностях, объясняется связь этого контраста с проводимостью образцов. С точки зрения развития метода DEBW достоинством низковольт- 
ной SEM-микроскопии является возможность быстрой и неразрушающей визуализации структур непосредственно после их записи электронным лучом. Полученные результаты могут быть информативны в качестве оценки метода низковольтной SEM-микроскопии для исследования и визуализации сегнетоэлектрических доменов в целом.

\section{2. Методы формирования и особенности визуализации доменных структур}

Формирование доменов проводилось в SEM марки JSM-840A с управляемым перемещением электронного луча с помощью программы NanoMaker. Исследовались номинально чистые образцы LN конгруэнтного состава (CLN) и образцы LN после восстановительного отжига $\left(T=550^{\circ} \mathrm{C}\right)$ в атмосфере водорода (RLN). As-grown кристаллы CLN имеют очень низкую проводимость $\left(G_{\mathrm{CLN}}=10^{-16}-10^{-18} \Omega^{-1} \mathrm{~cm}^{-1}\right)$. Согласно [24], используемый режим восстановительного отжига приводит к возрастанию проводимости до величин $G_{\mathrm{RLN}}=10^{-11}-10^{-13} \Omega^{-1} \mathrm{~cm}^{-1}$.

Домены формировались при локальных (условно точечных) облучениях полированных пластин толщиной $\sim 0.5 \mathrm{~mm}$ с кристаллографической ориентацией (010) и (001) ( $Y$ - и $Z$-срезы соответственно). Соответствующие схемы роста доменов на $Y$ - и $Z$-срезах показаны на рис. $1, a, b$. При облучении неполярного $(Y)$ среза домены, зарождающиеся в точке облучения, растут в поверхностном слое в направлении $+Z$ под действием тангенциальной составляющей поля пространственного заряда $E_{z}(r)$; толщина поверхностного слоя определяется энергией облучающих электронов [25]. Доменные решетки в $Y$-срезах записывались путем локальных облучений поверхности при перемещении луча вдоль $X$ и $Z$-направлений [25]. При облучении полярного $(-Z)$ среза домены растут вглубь кристалла. Доменные решетки в Z-срезах записывались путем создания системы локальных облучений поверхности при перемещении луча вдоль $Y$ - и $X$-направлений [26].

Принцип визуализации доменов в низковольтном режиме SEM на поверхности высокоомного сегнетоэлектрика [13] иллюстрируется рис. 1, $c$, на котором схематично представлена зависимость электронной эмиссии $(\sigma)$ из поверхностного слоя диэлектрика от ускоряющего напряжения $U$ в SEM. Как известно $[27,28]$, при $U=U_{2}$ количество вылетающих из образца вторичных (SE) (коэффициент эмиссии $\delta=I_{\mathrm{SE}} / I$ ) и отраженных (BSE) электронов (коэффициент эмиссии $\left.\eta=I_{\mathrm{BSE}} / I\right)$ равно количеству электронов, вносимых электронным лучом $\left(\sigma=\left(I_{\mathrm{SE}}+I_{\mathrm{BSE}}\right) / I \sim 1\right)$; образец при этих условиях облучения практически не заряжается. В условиях, соответствующих значениям $\sigma<1$, на поверхности накапливается отрицательный заряд, а при $\sigma>1$ - положительный.

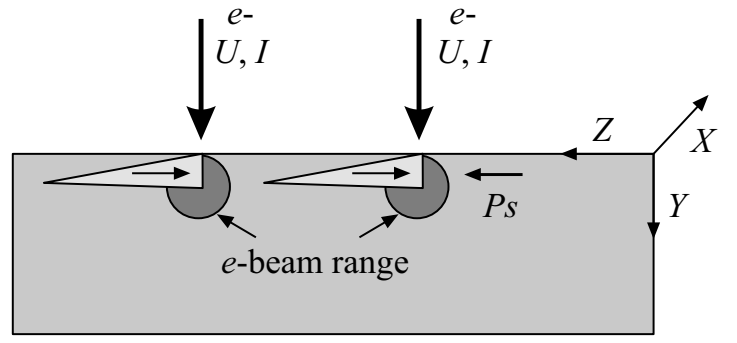

$a$

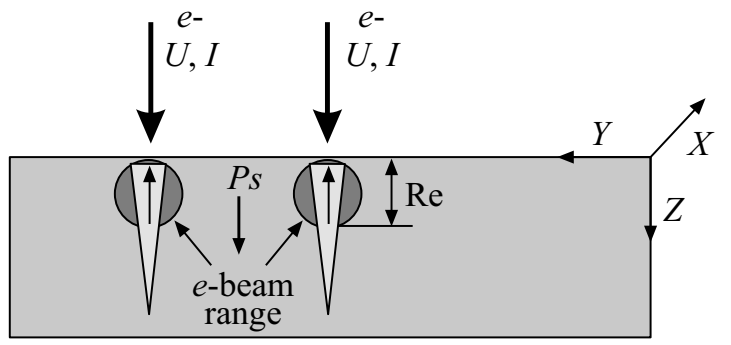

$b$

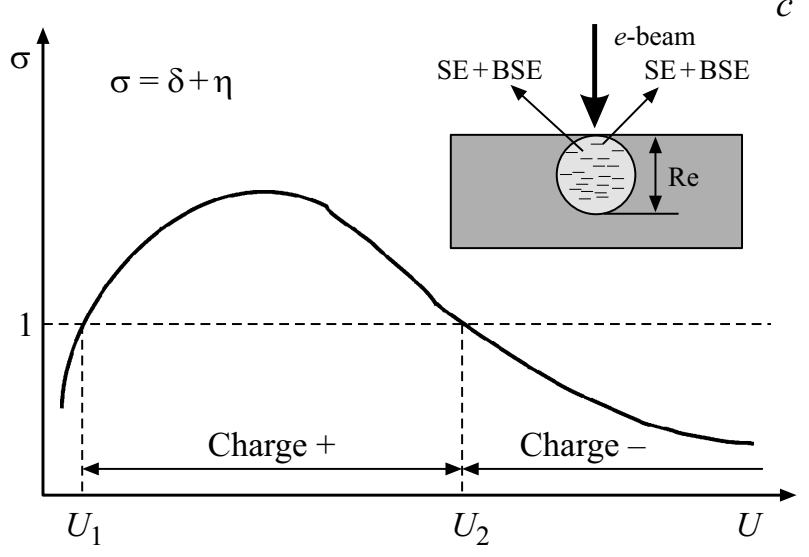

Рис. 1. Схема формирования и роста доменов при локальных облучениях поверхности LN: $a-Y$-срез, $b-Z$-срез; $c-$ условное представление изменения поверхностной электронной эмиссии $(\sigma)$ от ускоряющего напряжения микроскопа $(U)$; на вставке - схема внедрения зарядов электронным лучом.

Наиболее благоприятный режим наблюдения доменов как в CLN, так и RLN образцах соответствовал $U=2 \mathrm{kV}$. Это значение находится вблизи второй равновесной точки $\left(U_{2}\right)$, на экспериментальной зависимости $\sigma(U)$, определенной для $\mathrm{LN}$ [29].

B CLN визуализация доменов осложнялась чрезмерной отрицательной зарядкой поверхности. При записи доменных структур на больших участках поверхности и при использовании высоких ускоряющих напряжений часто возникал эффект „электронного зеркала“ (отражение электронного луча заряженной поверхностью) [30]. Для устранения поверхностного отрицательного заряда образец либо выдерживался в течение нескольких десятков или сотен минут без облучения, либо поверхность сканировалась электронным лучом при $U<U_{2}$. В последнем случае происходит переход к режиму облучения, соответствующему $\sigma>1$, и на поверхности начинают накапливаться положительные заряды, компенсирую- 

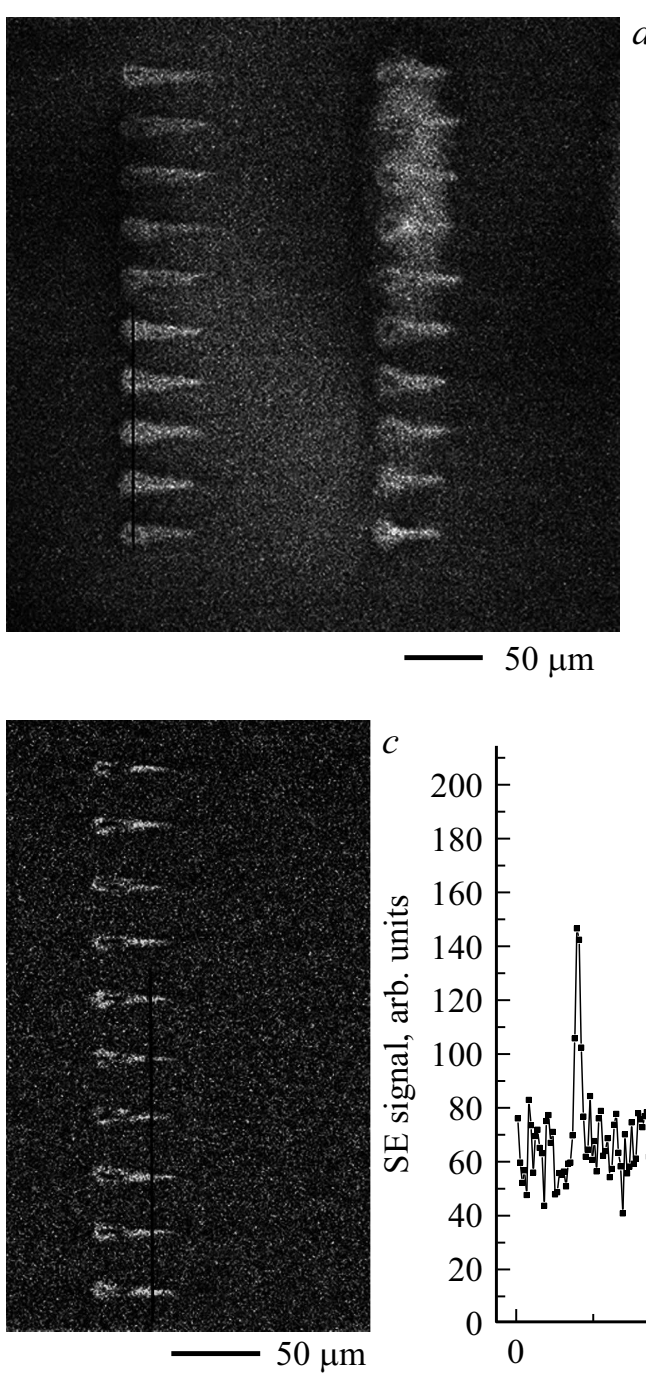
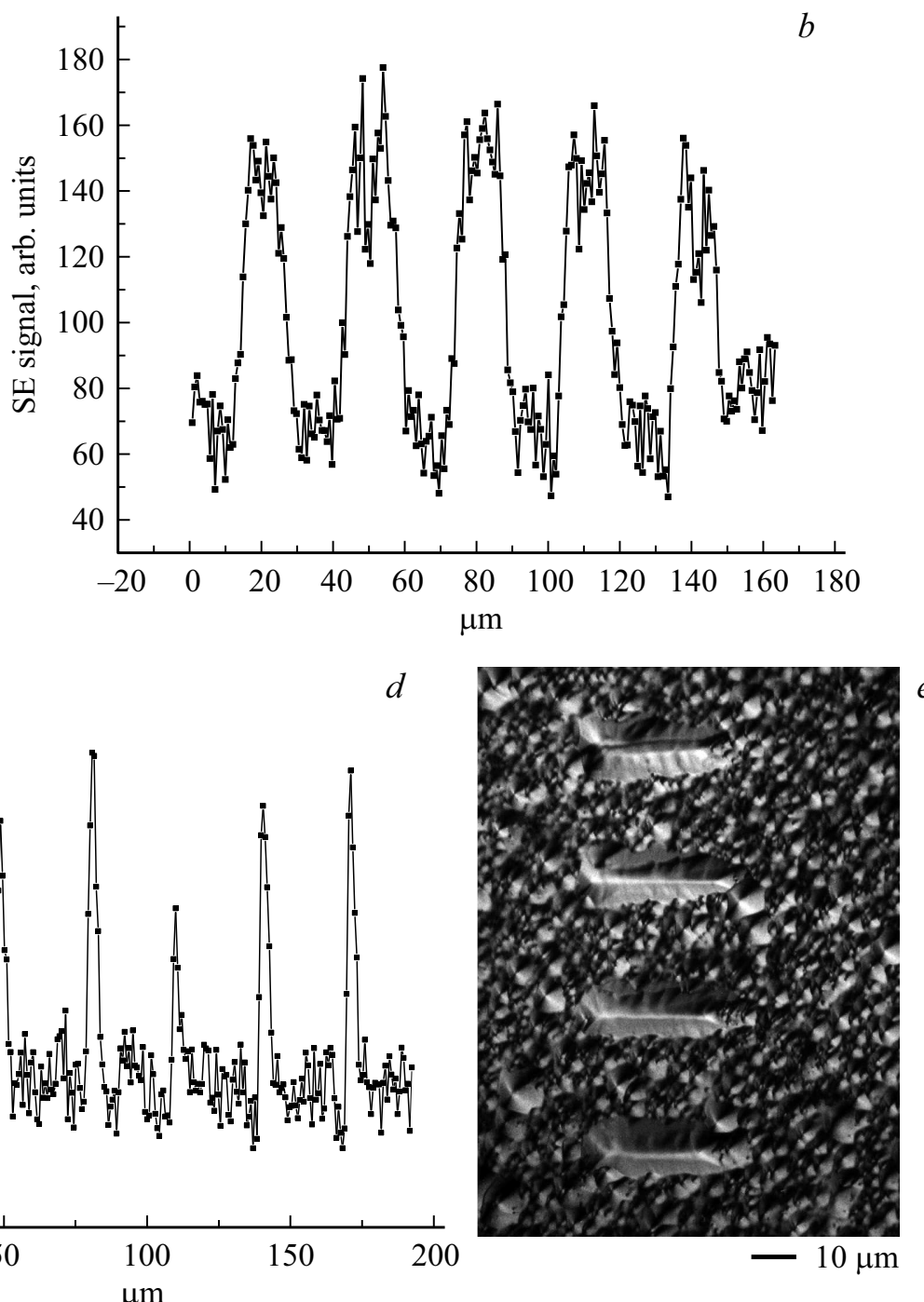

Рис. 2. Изображения доменов на $Y$-срезе CLN: потенциальное изображение и профиль изменения сигнала SE вскоре после DEBW $(a, b)$ и те же образцы спустя месяц после DEBW $(c, d)$; оптическое изображение доменов после химического травления $(e)$. Условия записи доменов: $U=15 \mathrm{kV}, t_{\text {irr }}=200 \mathrm{~ms}$.

щие заряд отрицательного знака (см. рис. 1,c) [27,28]. Устранение избыточного поверхностного заряда можно было ускорить и внешним воздействием, например, смачиванием поверхности этанолом.

В кристаллах RLN признаки зарядки поверхности после записи доменов не наблюдались, поскольку время диэлектрической релаксации (определяющее распад заряда) $\tau_{M}=\varepsilon \varepsilon_{0} / G$ составляет в RLN десятки или сотни s, что сопоставимо с временем перехода от записи доменов к низковольтному режиму для просмотра поверхности. Напротив, в CLN $\tau_{M} \geq 10^{4}-10^{6} \mathrm{~s}$, что обеспечивает довольно длительные времена сохранения зарядов после записи.

Для сопоставления с SE-изображениями домены в CLN- и RLN-образцах выявлялись методом селективного химического травления в смеси кислот $\mathrm{HF}+2 \mathrm{HNO}_{3}$. Так как отрицательные $(-Y$ и $-Z)$ поверхности травятся значительно быстрее, чем $+Y$ и $+Z$, домены выглядят холмиками или ямками, соответственно, на - и + срезах кристалла [31]. Изображения доменов после травления были получены в оптическом интерференционном микроскопе Axioplan 2.

\section{3. Экспериментальные результаты}

Ниже представлены и обсуждены изображения доменов и структур, полученные в режиме SE и после травления образцов.

\section{1. Характеристики доменов, записанных на $Y$ - и Z-срезах кристаллов CLN}

На рис. 2 показаны SE-изображения доменов на $Y$-срезе, зарегистрированные через сутки (рис. 2, $a, b)$, и через месяц (рис. 2,c,d) после записи; рядом показаны 


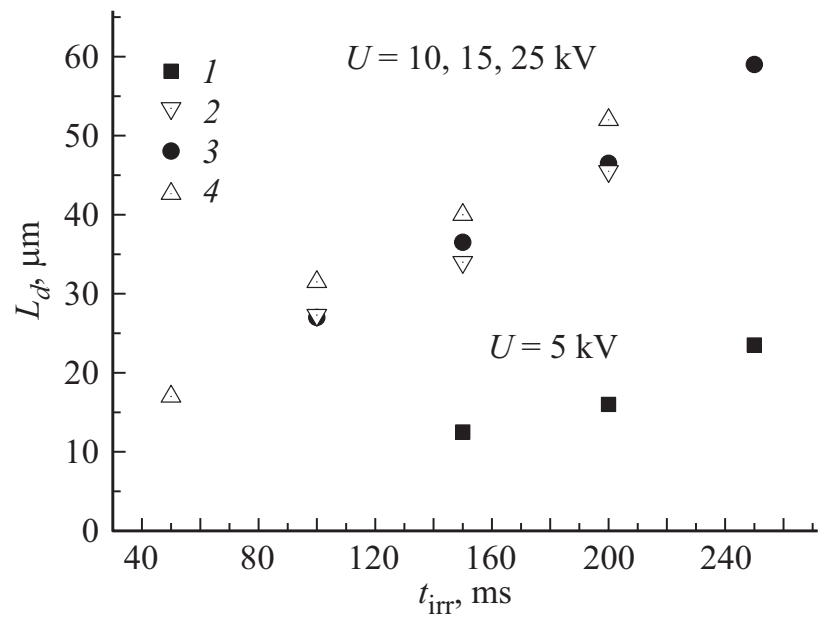

Pис. 3. Средняя длина потенциальных изображений доменов, созданных при разных ускоряющих напряжениях в SEM: $U=5 \mathrm{kV}(1), 10 \mathrm{kV}(2), 15 \mathrm{kV}(3), 25 \mathrm{kV}(4)$.

соответствующие профили сигнала SE. На рис. 2,e представлены те же домены, выявленные химическим травлением. На $Y$-срезе домен после травления имеет вид вытянутой, лежащей на одной из граней треугольной пирамиды, сужающейся к концу домена. Длина доменов на картинах травления хорошо согласуется с оценками длины на SE-изображениях.

Отметим характерные особенности SE-изображений доменов на $Y$-срезах. Как зона облучения (широкая овальная часть), так и область собственно домена ( вытянутая часть) характеризуются повышенным сигналом $\mathrm{SE}$ (светлый контраст на рис. 2, $a, c)$; при этом в центре области облучения сигнал SE несколько понижен по сравнению с краями (рис. 2, $b$ ). По профилям изменения сигналов SE была сделана оценка размеров SE-изобра-
Диаметр SE-изображений областей облучения на $Y$-срезе

\begin{tabular}{c|c|c|c|c}
\hline $\begin{array}{c}\text { Режимы } \\
\text { облучения } \\
\left(S_{\text {irr }}=0.5 \mu \mathrm{m}^{2}\right)\end{array}$ & $U=25 \mathrm{kV}$ & $U=15 \mathrm{kV}$ & $U=10 \mathrm{kV}$ & $U=5 \mathrm{kV}$ \\
\hline$t_{\text {irr }}=50 \mathrm{~ms}$ & $11.1 \mu \mathrm{m}$ & $9.8 \mu \mathrm{m}$ & - & - \\
$t_{\text {irr }}=100 \mathrm{~ms}$ & $13.5 \mu \mathrm{m}$ & $11.9 \mu \mathrm{m}$ & $11.1 \mu \mathrm{m}$ & $9.2 \mu \mathrm{m}$ \\
$t_{\text {irr }}=150 \mathrm{~ms}$ & $14 \mu \mathrm{m}$ & $12.1 \mu \mathrm{m}$ & &
\end{tabular}

жения овальной области облучения в зависимости от экспериментальных условий записи доменов ( $U$ и $t_{\text {irr }}$ при постоянном токе $I=0.1 \mathrm{nA})$. Измерялась ширина на полувысоте соответствующего изменения интенсивности сигнала SE. Результаты измерений, выполненные после записи доменов спустя сутки, представлены в таблице.

Как следует из таблицы, диаметр SE-изображений намного превышает размеры реальной зоны облучения. Зона локального облучения расширяется при увеличении ускоряющего напряжения $U$ и времени облучения $t_{\text {irr }}$. Спустя месяц диаметр SE-изображения зоны облучения и ширина доменов уменьшились примерно в $1.5-2$ раза (рис. $2, c, d$ ). Напротив, длина $L_{d}$ доменов практически не изменилась в течение месяца. На рис. 3 представлены зависимости $L_{d}$ от условий записи, измеренные по

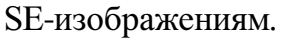

Рис. 4, $a$ демонстрирует SE-изображение поверхности после записи доменов на $-Z$-срезе кристаллов CLN; рядом (рис. $4, b)$ представлен соответствующий профиль сигнала SE вдоль линии, пересекающей характерные участки. На рис. 4, с показана эта же структура после травления образца. Как и на неполярном срезе, область облучения характеризуется повышенным сигналом SE (светлый контраст). Анализ нескольких изображений по-
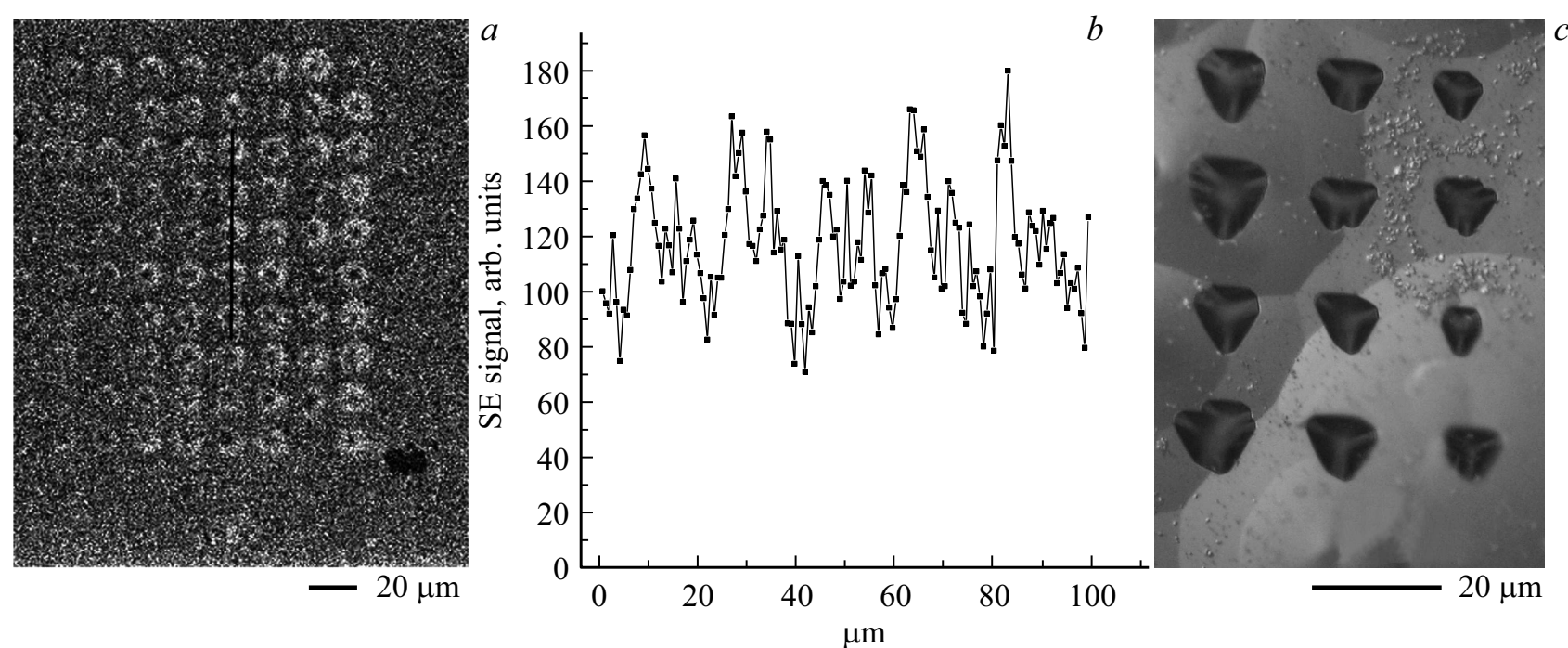

Рис. 4. SE-изображение поверхности -Z-среза кристалла CLN после DEBW, $U=25 \mathrm{kV}-(a)$; профиль изменения сигнала $\mathrm{SE}$ вдоль линии, пересекающей характерные зоны SE-изображения - $(b)$; оптическое изображение созданных доменов после химического травления - $(c)$. 


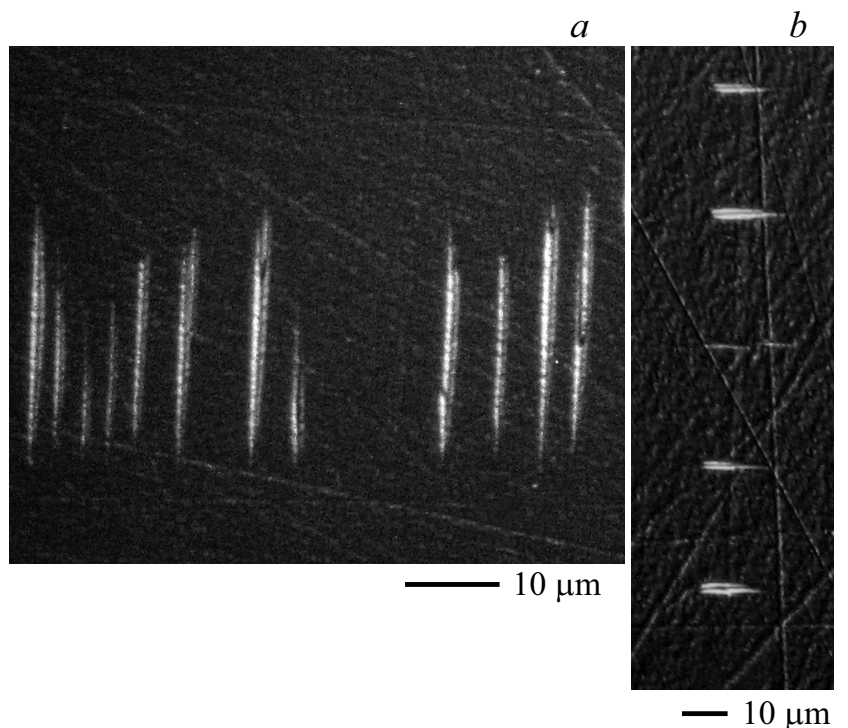

Рис. 5. Оптические изображения доменов, созданных в RLN-кристалле на $Y$-срезе после химического травления образца: (a) - расстояние между облучениями $\Lambda=4 \mu \mathrm{m}$ и (b) $-\Lambda=30 \mu \mathrm{m}$ казал, что размеры SE-изображений облученных участков на Z-срезе, так же как и на Y-срезе, расширяются с

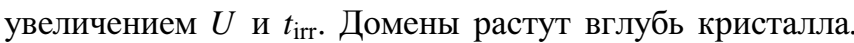
Пониженный сигнал SE в центре зоны облучения, возможно, связан с возникновением доменов.

\section{2. Характеристики доменов, записанных на $Y$ - и Z-срезах кристаллов RLN}

Формирование доменов в кристаллах восстановленного ниобата лития (RLN) потребовало использования более „жестких“ режимов электронного облучения, чем в CLN [32]. Домены, записанные на неполярных $Y$-срезах, не выявлялись в режиме SE и были обнаружены только методом химического травления. Пример картин травления участков с доменами показан на рис. 5, $a, b$.

В отличие от неполярного среза, SE-изображения доменных структур, созданных электронным лучом на Z-срезе, были выявлены. Характерное SE-изображение и соответствующий профиль изменения сигнала SE для одной из структур показаны на рис. $6, a, b$. Рядом представлено оптическое изображение после травления той
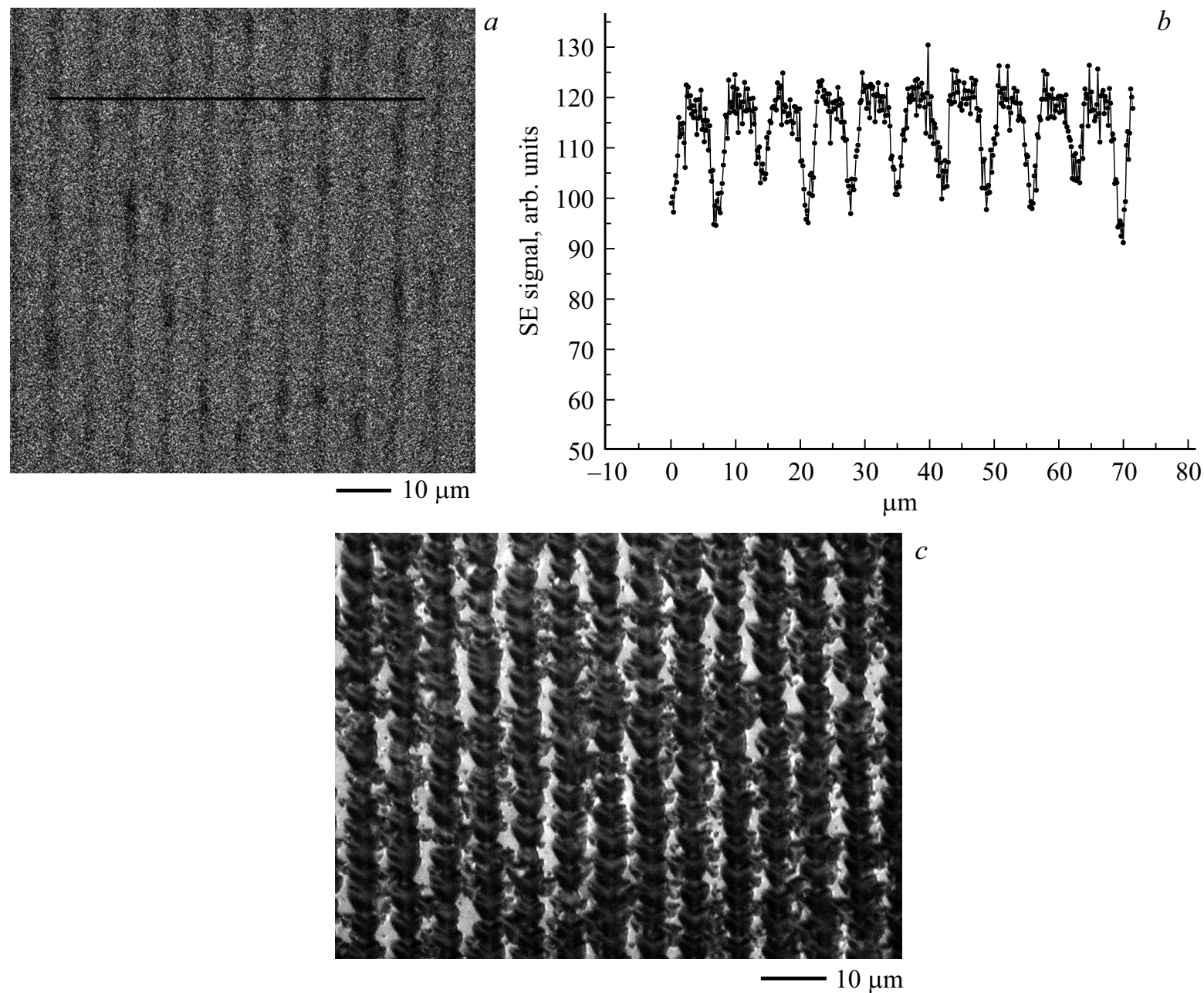

Рис. 6. SE-изображение доменной решетки $(\Lambda=7 \mu \mathrm{m})$, созданной на $-Z$-срезе образца RLN $-(a)$; профиль изменения сигнала $\mathrm{SE}$ поперек доменных линий решетки - $(b)$; оптическое изображение той же решетки после химического травления - $(c)$. 
же структуры. Как следует из картин травления, ряды доменной решетки сформированы близко расположенными по отношению друг к другу доменами треугольной формы, растущими вглубь образца. Важно подчеркнуть, что в отличие от CLN, потенциальные изображения сформированных электронным лучом доменных линий характеризуются понижением сигнала SE по отношению к необлучаемой матрице. Вторичные электроны вылетают из достаточно тонкого слоя поверхности, поэтому ширина доменных линий на SE-изображении немного уже, чем в травленом образце, открывающем структуру после удаления достаточно толстого поверхностного слоя.

\section{4. Обсуждение результатов}

Низкоэнергетичные вторичные электроны, вылетающие из тонкого поверхностного слоя кристалла (в диэлектриках $\sim$ сотни $\AA$ [33]), обычно очень чувствительны к распределению электрических полей в образце. При сканировании электронным лучом, на поверхности гладких полированных образцов может возникать контраст, связанный с изменением количества SE, детектируемых в SEM. Такой вид контраста называют потенциальным или вольтовым. В зависимости от причин формирования существует несколько видов потенциального контраста [34]. Если потенциал облучаемой поверхности влияет на энергию подлетающих первичных электронов, то возникает потенциальный контраст I типа. При этом, изменение числа $\mathrm{SE}$ связано с зависимостью $\sigma(U)$ (см. pис. $1, c)$. Очевидно, что I тип потенциального контраста полностью отсутствует в RLN-кристаллах, т. к. заряд в этих образцах распадается еще до начала исследования доменов. Избыточная зарядка CLN-образцов, влияющая на энергию подлетающих первичных электронов, обычно перед исследованием доменов устранялась. Поэтому эта разновидность контраста в дальнейшем не будет учитываться.

Потенциальный контраст II типа возникает из-за влияния на SE электрических полей в образце. К этому типу контраста относится, например, контраст микросхем [34]. Положительное поле в образце уменьшает число SE, а отрицательное увеличивает. Потенциальный контраст III типа зависит от тангенциальных компонент электрических полей, которые, отклоняя SE, влияют на четкость детектируемых изображений. Отдельно выделяется потенциальный контраст, связанный с существованием спонтанной электрической поляризации $\left(P_{s}\right)$ и ее влиянием на SE в сегнетоэлектрических доменах. Этот потенциальный контраст называется потенциальным контрастом IV типа [34].

Обсудим в рамках этих представлений причины и особенности появления потенциального контраста SE-изображений доменов в CLN и RLN-кристаллах.

Формирование доменов в обоих типах исследуемых кристаллов происходило в результате интенсивной локальной накачки отрицательных зарядов (диапазон $U=5-25 \mathrm{kV}$, соответствует условию $\sigma<1)$.
В результате в образце на глубине вплоть до нескольких микрон возникает область отрицательного пространственного заряда $\left(Q_{\mathrm{sc}}\right)$. Поле этого заряда $\left(E_{\mathrm{sc}}\right)$ переключает спонтанную электрическую поляризацию при условии $E_{\mathrm{sc}}>E_{\mathrm{c}}$ (коэрцитивное поле $\left.\mathrm{LN}-E_{\mathrm{c}}=2.2 \cdot 10^{7} \mathrm{~V} / \mathrm{m}[35]\right)$.

Изображения сформировавшихся доменов были получены после прекращения облучений, когда внедряемый лучом заряд начинает распадаться. Кинетику распада заряда $Q_{\mathrm{sc}}$ после завершения процесса записи обычно представляют выражением [36]:

$$
Q(t)=Q_{\mathrm{sc}} \exp \left(-t / \tau_{M}\right)
$$

Из-за разной проводимости исследованных кристаллов процесс распада отрицательного заряда в CLN и RLN протекал с разной скоростью.

В кристаллах CLN из-за отклонения состава от стехиометрического и дефицита $\mathrm{Li}$ транспорт заряда на микроскопическом уровне определяется внутренним собственным структурным дефектом $\mathrm{Nb}_{\mathrm{Li}}$ (Nb-antisites) [35]. Этот тип собственного дефекта является основным центром захвата электронов, способным сохранять заряд длительное время. В кристаллах RLN в результате умеренного восстановительного отжига формируются другие структурные дефекты, так называемые биполяроны $\left(\mathrm{Nb}_{\mathrm{Nb}}-\mathrm{Nb}_{\mathrm{Li}}\right)^{2-}$, что, в результате, меняет схему транспорта заряда. Вместо захвата электронов ловушкой $\mathrm{Nb}_{\mathrm{Li}}$ происходит их захват ловушкой $\mathrm{Nb}_{\mathrm{Nb}}$ и формирование нестабильного полярона малого радиуса $\mathrm{Nb}_{\mathrm{Nb}}^{4+}$. Такая схема транспорта заряда в RLN и приводит к улучшению проводящих свойств этого кристалла [35].

Из-за разных типов ловушек кинетику распада внедренного лучом заряда представим как в [37], в виде быстрой и медленной стадии разрядки

$$
Q(t)=Q_{\mathrm{tr}}+\Delta Q \exp (-t / \tau) .
$$

Здесь $Q_{\mathrm{tr}}-$ часть заряда $Q_{\mathrm{sc}}$, сохраняющаяся в зоне облучения на некоторой глубине в течение очень длительного времени благодаря захвату на глубокие ловушки $\left(\mathrm{Nb}_{\mathrm{Li}}\right) ; \Delta Q$ - заряд, осевший на неглубоких и поверхностных уровнях; $\tau$ - постоянная разрядки, зависящая от свойств материала. В облученных кристаллах RLN преобладает быстрая составляющая разрядки, что не позволило обнаружить следы зарядки после выключения электронного луча. В кристаллах $\mathrm{CLN}$, напротив, из-за большого количества характерного для конгруэнтных образцов структурного дефекта $\mathrm{Nb}_{\mathrm{Li}}$ распад заряда, по-видимому, происходит в виде двух стадий [37]. После относительно быстрой разрядки неглубоких и поверхностных уровней, доля заряда $Q_{\mathrm{tr}}$ сохраняется очень долго и может служить причиной возникновения в зоне записи электронным лучом потенциального контраста II типа. Сохранившиеся заряды в зоне облучений могут создавать внутри диэлектрика локальное отрицательное поле, усиливающее выход SE из этого участка. На $Y$-срезах светлый SE-контраст формируется не только в зоне 
непосредственного облучения, но и в области доменов (рис. 2). Одним из объяснений появления такого контраста на доменах является возможное окружение обычно наклонных доменных стенок облаком зарядов. Судя по экспериментальным результатам, детектируемые размеры зон облучения и ширина шлейфа зарядов, декорирующих домены, существенно уменьшаются спустя месяц после первого просмотра. Видимо, это происходит из-за постепенного распада заряда $Q_{\mathrm{tr}}$. За месяц ширина SE-изображений доменов уменьшилась и стала сопоставимой ширине доменов, определенной ранее методом латеральной РFM-микроскопии [38]. В хорошем согласии с измерениями, выполненными ранее методом PFM [25,38], находятся и представленные на рис. 3 длины доменов. Это говорит о соответствии обнаруженного потенциального контраста II типа на доменных структурах в кристаллах CLN c реальной доменной структурой, сформированной электронным лучом.

В отличие от кристаллов CLN, SE-изображения доменов в RLN-образцах обнаружены только на полярных $Z$-срезах. Причем контраст доменов формировался из-за снижения сигнала SE в области доменных линий, которые являются положительными доменами, по отношению к остальной части кристалла. Полярность доменов в данном случае подтверждена картинами травления (см. рис. $6, c$ ). Полученный результат совпадает с ранними результатами по исследованию SE-изображений доменов в SEM [13-15]. Если бы изображение доменов разной полярности возникало в результате локального нагрева электронным лучом, то положительные домены должны были иметь более светлое изображение, чем отрицательные, как достоверно показано в [29]. Кроме того, отсутствие каких-либо эффектов зарядки при сканировании электронным лучом по поверхности кристалла RLN говорит о невозможности применения моделей контраста, использованных в [17-23]. Следовательно, наблюдаемый нами доменный контраст вызван не нагревом и не зарядкой электронным лучом, а только влиянием спонтанной электрической поляризации на выход $\mathrm{SE}$ из поверхностного слоя доменов. Домены с положительным направлением вектора $P_{s}$ имеют пониженный сигнал SE. Отметим, что такой тип потенциального контраста невозможен на $Y$-срезах, когда вектор $P_{s}$ параллелен поверхности, что подтверждается нашими исследованиями.

Таким образом, более высокая проводимость кристаллов RLN позволила обнаружить еще один тип потенциального контраста - потенциальный контраст IV типа, или контраст сегнетоэлектрических доменов. После формирования методом DEBW изображения PPLN в SEM хорошо соответствовали параметрам и размерам этих же участков кристалла, выявленных химическим травлением.

\section{5. Заключение}

Детальное рассмотрение потенциальных SEM-изображений структур, созданных методом DEBW, показало, что природа выявленного потенциального контраста различна в образцах ниобата лития с разной проводимостью. В высокоомных кристаллах CLN на $Z$ - и $Y$-срезах, независимо от направления вектора $P_{s}$, отрицательные заряды, локализованные в зоне записи на глубоких электронных ловушках, создают в диэлектрике поле, увеличивающее сигнал SE. В результате, при исследовании в режиме SE может возникать потенциальное изображение, соответствующее созданным электронным лучом доменным структурам. Такой тип потенциальных изображений позволяет исследовать взаимосвязь между режимами DEBW и локализацией и распределением электронных зарядов в облучаемых участках и в области сформировавшихся доменов. По потенциальным изображениям на $Y$-срезах можно достоверно определять размеры созданных доменов и контролировать регулярность и периодичность сформированной области PPLN.

В более проводящих кристаллах RLN при исследовании обнаруживается потенциальный контраст, зависящий от направления спонтанной поляризации доменов - сегнетоэлектрический контраст. Детектируемый сигнал SE с поверхности положительных доменов ниже, чем сигнал SE отрицательной матрицы. В $Y$-срезах вектор $P_{s}$ параллелен поверхности и домены в SEM не визуализировались. Данный результат полезен для понимания природы потенциального контраста сегнетоэлектрических доменов в целом.

Автор благодарит за помощь в написании статьи профессора ИК РАН Т.Р. Волк.

\section{Список литературы}

[1] P. Ferraro, S. Grilli, P.D. Natale. Ferroelectric Crystals for Photonic Applications. Springer-Verlag, Berlin, Heidelberg (2009).

[2] J. He, S.H. Tang, Y.Q. Qin, P. Dong, H.Z. Zhang, C.H. Kang, W.X. Sun, Z.X. Shen. J. Appl. Phys. 93, 9943 (2003).

[3] M. Fujimura, K. Kintaka, T. Suhara, H. Nishihara. J. Light. Tech. 11, 1360 (1993).

[4] C. Restoin, S. Massy, C. Darraud-Taupiac, A. Barthelemy. Opt. Mater. 22, 193 (2003).

[5] J. Son, Y. Yuen, S. Orlov, L. Hesselink. J. Cryst. Growth 281, 492 (2005).

[6] P. Molina, M.O. Ramirez, J. Garcia-Sole, L.E. Bausa. Opt. Mater. 31, 1777 (2009).

[7] L. Mateos, L.E. Bausa, M.O. Ramirez. Appl. Phys. Lett. 102, 042910 (2013).

[8] L.S. Kokhanchik, M.V. Borodin, N.I. Burimov, S.M. Shandarov, V.V. Shcherbina, T.R. Volk. IEEE TUFFC 59, 1076 (2012).

[9] T.R. Volk, L.S. Kokhanchik, R.V. Gainutdinov, Ya.V. Bodnarchuk, S.M. Shandarov, M.V. Borodin, S.D. Lavrov, H. Liu, F. Chen. J. Lightwave Technology 33, 4761 (2015).

[10] D.S. Chezganov, E.O. Vlasov, M.M. Neradovskiy, L.V. Gimadeeva, E.A. Neradovskaya, M.A. Chuvakova, H. Tronche, F. Doutre, P. Baldi, M.P. De Micheli, V.Ya. Shur. Appl. Phys. Lett. 108, 192903 (2016).

[11] E. Soergel. Appl.Phys. B 81, 729 (2005). 
[12] R. Prashant Potnis, Nien-Ti Tsou, John E. Huber. Materials 4, 417 (2011).

[13] R. Le Bihan. Ferroelectrics 97, 19 (1989).

[14] R. Le Bihan, M. Maussion. Ferroelectrics 7, 307 (1974).

[15] S. Ikeda, Y. Uchikawa. J. Electron Microscopy 29, 209 (1980).

[16] G. Rosenman, A. Skliar, I. Lareah, N. Angert, M. Tseitlin, M. Roth. Phys. Rev. B 54, 6222 (1996).

[17] S. Zhu, W. Cao. Phys. Status Solidi A 173, 495 (1999).

[18] V.V. Aristov, L.S. Kokhanchik, V.G. Monya. Ferroelectrics 70, 15 (1986).

[19] A.A. Sogr. Ferroelectrics 97, 47 (1989).

[20] V.V. Aristov, L.S. Kokhanchik, K.-P. Meyer, K. Blumtritt. Phys. Status Solidi A 78, 229 (1983).

[21] L.S. Kokhanchik. Micron 40, 41 (2009).

[22] J. Li, H.X. Yang, H.F. Tian, C. Ma, S. Zhang, Y.G. Zhao, J.Q. Li. Appl. Phys. Lett. 100, 152903 (2012).

[23] J.M. Saldaña, J.M.C. Moreno, G.A. Schneider. Key Eng. Mater. 264-268, 1185 (2004).

[24] P.F. Bordui, D.H. Jundt, E.M. Standifer, R.G. Norwood, R.L. Sawin, J.D. Galipeau. J. Appl. Phys. 85, 3766 (1999).

[25] L.S. Kokhanchik, R.V. Gainutdinov, S.D. Lavrov, E.D. Mishina, T.R. Volk. Ferroelectrics 480, 49 (2015).

[26] L.S. Kokhanchik, M.N. Palatnikov, O.B. Shcherbina. Phase Transitions 84, 797 (2011).

[27] J. Cazaux. J. Appl. Phys. 85, 1137 (1999).

[28] Микроанализ и растровая электронная микроскопия / Под ред. Ф. Морис, Л. Мени, Р. Тиксье / Пер. с франц. под ред. И.Б. Боровского. Металлургия, М. (1985). 392 с.

[29] K. Tanaka, T. Suhara. Electronics Lett. 51, 923 (2015).

[30] J.P. Vigouroux, J.P. Duraud, A. Le Moel, C. Le Gressus, D.L. Griscorn. J. Appl. Phys. 57, 3195 (1985).

[31] O. Norio, I. Takashi. J. Appl. Phys. 46, 1063 (1975).

[32] L.S. Kokhanchik, Ya.V. Bodnarchuk, T.R. Volk. J. Appl. Phys. 122, 104105 (2017).

[33] И.М. Бронштейн, Б.С. Фрайман. Вторичная электронная эмиссия. Наука, М. (1969) 408 с.

[34] H. Seiler. J. Appl. Phys. 54, R1 (1983).

[35] T. Volk, M. Woehlecke. Lithium niobate: defects, photorefraction and ferroelectric switching. Springer-Verlag, Berlin, Heidelberg (2008). 247 p.

[36] J. Cazaux. J. Appl. Phys. 59, 1418 (1986).

[37] S. Fakhfakh, O. Jbara, S. Rondot, A. Hadjadj, J.M. Patat, Z. Fakhfakh. J. Appl. Phys. 108, 093705 (2010).

[38] L.S. Kokhanchik, R.V. Gainutdinov, S.D. Lavrov, T.R. Volk. J. Appl. Phys. 118, 072001 (2015).

Редактор Ю.Э. Китаев 